\title{
Ziv-Zakai Lower Bound for Impulse Radio Ultra-WideBand Ranging Error Correlation Matrix
}

\author{
Hai Zhan ${ }^{1}$, Jean-Yves Le Boudec ${ }^{1}$ and John Farserotu ${ }^{2}$ \\ ${ }^{1}$ LCA2, Ecole Polytechnique Fédérale de Lausanne (EPFL), CH-1015, Lausanne, Switzerland \\ ${ }^{2}$ Centre Suisse d'Electronique et de Microtechnique S.A.,Jaquet Droz 1, CH-2007, Neuchatel, Switzerland \\ Email: \{hai.zhan, jean-yves.leboudec\}@epfl.ch \\ john.farserotu@csem.ch
}

\begin{abstract}
We derive the Ziv-Zakai lower bound for Impulse Radio (IR) Ultra-WideBand (UWB) ranging error correlation matrix under a classical IR-UWB positioning system. We present the numerical evaluations with IEEE 802.15.4a channel model and the geometry of indoor environments of interest. As our derived bound depends on the geometry of the indoor environments, our bound can be used in real environments with the channel measurements from real environments.
\end{abstract}

\section{INTRODUCTION}

Impulse Radio (IR) Ultra-WideBand (UWB) can be used for precise localization applications [1]. Determination of ranging accuracy is a fundamental problem. Several previous work discuss the lower bound minimum Mean Square Error (MSE) of UWB ranging system. Zhang et al. derive the Cramér-Rao Lower Bound (CRLB) for the time-delay estimation of UWB signals in [2]. Due to Ziv-Zakai lower bound is more accurate than CRLB under moderate and low SNR [3], Hicham et al. also derive a Ziv-Zakai time delay estimation bound for UWB signals in [4]. Dardari et al. derive a Ziv-Zakai lower bound for the IR-UWB ranging error using both the IEEE 802.15.4a channel models and experimentally measured data in [5]. However, the above work do not consider the lower bounds of ranging error correlations among the different base stations of a UWB positioning system.

In contrast to the previous work, we derive the Ziv-Zakai lower bound of the ranging error correlation matrix under a classical IR-UWB positioning system. Through the numerical evaluation results, we prove that the ranging errors among the different base stations are correlated. As our derived bound depends on the geometry of the indoor environments, our bound can be used in real environments with the channel measurements from real environments. Our work is fundamental for Time-of-Arrived (TOA) based positioning or TimeDifference-of-Arrived based positioning.

The paper is organized as follows: We explain our problem in Section II. In Section III, we discuss the statistical properties of random parameters. We derive Ziv-Zakai lower bound for IR-UWB ranging error correlation matrix in Section IV. We present numerical evaluations in Section V. And we give conclusions in Section VI.

\section{Problem Statement}

We consider a network with a processing center, $I$ Base Stations (BS) and multiple Mobile Tags (MT). The MTs transmit one IR-UWB signal to the BSs. The processing center uses the received signals of the BSs to estimate the positions of MTs. All base stations are perfectly synchronized by sharing the same local clock through a cable connection. All base stations are positioned at known coordinates in the area to be monitored. The channels between the base stations and the mobile tag are assumed to be dense multipaths propagation environments. The MTs' positions are estimated independently, and hence we can focus on one MT in the network without loss of generality.

The transmitter transmits an IR-UWB transmitted signal $f(t)$ to the receiver. Given the sampling interval is $T_{s}$, the transmit time of the mobile tag is $T_{\eta} \simeq \eta T_{s}$, where $\eta \in \mathbb{Z}$ and $\eta \geq 0$ (as shown in Fig.1). We define the discrete transmit pulse as $\vec{f}=\left[f(0), \cdots, f(u), \cdots, f((U-1)]^{T}\right.$, $u \in\{0, \cdots, U-1\}, T_{f}=U T_{s}$ and $U \in \mathbb{Z}$. The superscript $T$ denotes the transpose operator.

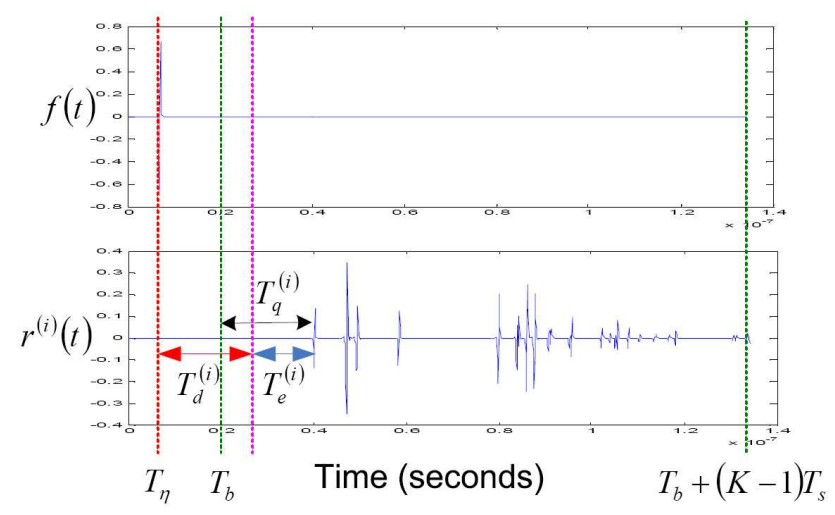

Fig. 1. The transmitted signal and received signal of the base station. $T_{\eta}$ is the transmit time of the mobile tag. The received signals are received during the observational interval $\left[T_{b}, T_{b}+(K-1) T_{s}\right]$. This figure only shows $T_{b}>T_{\eta}$ case. The case of $T_{b} \leq T_{\eta}$ is also possible.

The $i$-th base station received $K$ samples concatenated by $\vec{r}^{(i)}=\left[r^{(i)}(0), \cdots, r^{(i)}(k), \cdots, r^{(i)}(K-1)\right]^{T}$ during the observational interval $\left[T_{b}, T_{b}+(K-1) T_{s}\right], k \in\{0, \cdots, K-1\}$ , $K \in \mathbb{Z}$ and $K>U$ (as shown in Fig.1).

Hence, $\vec{r}^{(i)}$ can be represented as following:

$$
\vec{r}^{(i)}=\Psi \vec{h}^{(i)}+\vec{z}^{(i)}=\vec{\rho}^{(i)}+\vec{z}^{(i)},
$$

where $\vec{\rho}^{(i)}=\Psi \vec{h}^{(i)}$ and $\vec{z}^{(i)} \in \mathbb{R}^{K}$ is an additive independent 
white Gaussian noise with zero mean and unknown variance $\left(\sigma^{(i)}\right)^{2} . \Psi=\left[\begin{array}{cccc}\vec{\psi}_{0} & \vec{\psi}_{1} & \cdots & \vec{\psi}_{(K-M)}\end{array}\right]$ is a $K \times(K-U+$ $1)$ dictionary matrix, which is generated by shifting with one sample of $\vec{f}$, leading to a set of parameterized waveforms given by $\vec{\psi}_{j}=\left[\begin{array}{lll}\overrightarrow{0}_{j}^{T} & \vec{f}^{T} & \overrightarrow{0}_{(K-U-j)}^{T}\end{array}\right]^{T}$, where $\overrightarrow{0}_{j}=[\underbrace{0, \cdots, 0}_{j}]^{T}$, and $\overrightarrow{0}_{(K-U-j)}=[\underbrace{0, \cdots, 0}_{K-U-j}]^{T}$, where $j \in\{0, \cdots, K-U\}$ and $\vec{h}^{(i)} \in \mathbb{R}^{K-U+1}$ is the channel impulse response between the mobile tag and $i$-th base station.

The distance between the mobile tag and $i$ th base station can be expressed as follows $d^{(i)}=\sqrt{\left(x-x^{(i)}\right)^{2}+\left(y-y^{(i)}\right)^{2}}$. And $\vec{g}$ is defined to be $\vec{g}=\left[g^{(1)}, \cdots, g^{(I)}\right]^{T}$, where $g^{(i)}=\left\lfloor\frac{d^{(i)}}{c \cdot T_{s}}\right\rfloor$ and $c$ is assumed to be $3 \cdot 10^{8} \mathrm{~m} / \mathrm{s}$. The range bias between the $i$-th BS and MT is $e^{(i)} \geq 0$ induced by Non-Line of Sight (NLOS) propagation.

As shown in Fig.1, $T_{q}^{(i)}$ can be computed as $T_{q}^{(i)}=T_{d}^{(i)}+$ $T_{\eta}+T_{e}^{(i)}-T_{b}$ for both $T_{b}>T_{\eta}$ and $T_{b} \leq T_{\eta}$ cases, where $T_{e}^{(i)}=\frac{e^{(i)}}{c}$. And $T_{b}$ should satisfy $0 \leq T_{b} \leq \min \left\{T_{\eta}+T_{d}^{(i)}\right\}$. Therefore, $\vec{h}^{(i)}=\left[\begin{array}{ccc}\overrightarrow{0}_{q^{(i)}}^{T} & \overrightarrow{0}_{b_{N L}^{(i)}}^{T} & \vec{\alpha}^{(i)^{T}}\end{array}\right]^{T}$, where $q^{(i)}=$ $\left\lfloor\frac{T_{q}^{(i)}}{T_{s}}\right\rfloor$. and $b_{N L}^{(i)}=\left\lfloor\frac{T_{e}^{(i)}}{T_{s}}\right\rfloor$.

The estimation problem is defined as:

\section{- Given:}

- the all BSs' received signals $\vec{r}^{(i)}$ and transmitted pulse $\vec{f}$, where $i \in\{1, \cdots I\}$,

- $T_{b}$ is known in practice,

- $\vec{\alpha}^{(i)}, T_{\eta}, e^{(i)}$ and $g^{(i)}$ are assumed to be random parameters,

- $\sigma^{(i)}$ is assumed to be a deterministic parameter.

\section{- Estimate:}

- compute the Ziv-Zakai lower bound of the ranging error correlation matrix.

\section{The Statistical Distributions of the Random PARAMETERS}

We assume the following issues:

- The random parameters, $\vec{g}, T_{\eta}, \vec{\alpha}^{(1)}, \cdots, \vec{\alpha}^{(I)}, e^{(1)}, \cdots$, $e^{(I)}$ are independent from each other.

- The Gaussian noise $\vec{z}^{(i)}$ are independent from each other, where $i \in\{1, \cdots I\}$.

- The distribution of $g^{(i)}$ depends on the geometry of indoor environments.

Example: There is a rectangular room with furniture (as shown in Fig.2). As the mobile tag can't come to the region where the furniture is placed and is only uniformly distributed in a non-furniture region, the position $(x, y)$ of the mobile tag is not uniformly distributed in the room. We use rejection sampling method [6] to obtain the samples of $(x, y)$ that are uniformly distributed in the nonfurniture region. Then we can obtain the corresponding samples of $g^{(i)}$. Finally, we obtain the pdf function with the histogram of $g^{(i)}$.

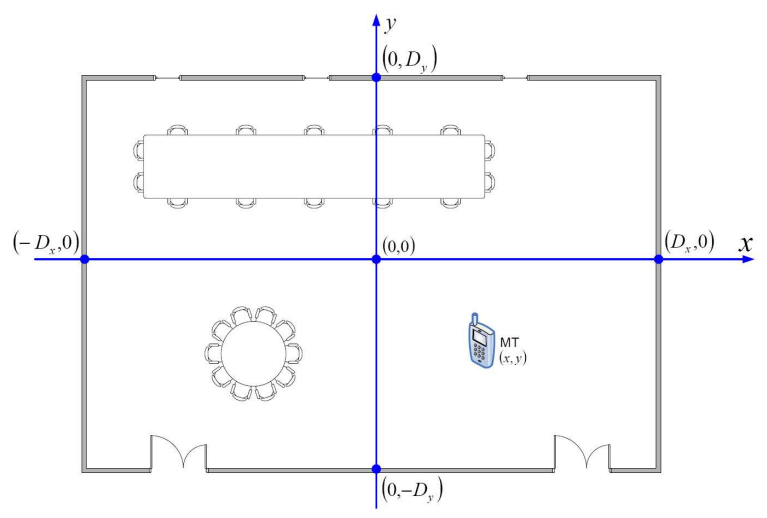

Fig. 2. A rectangular room with furniture.

- $T_{\eta}$ is assumed to be uniformly distributed between $\left[0, T_{\eta}^{\prime}\right]$ where $T_{\eta}^{\prime} \in \mathbb{R}$ and $T_{\eta}^{\prime} \geq 0$.

- We can obtain the distribution of $e^{(i)}$ by performing ranging measurements in the indoor environments of interest [7]. As the NLOS bias are always modeled independently (Eq.1 in [7]), we consider $e^{(i)}$ as a nuisance parameter in the derivation process of the Ziv-Zakai lower bound of ranging error correlation matrix.

- The prior distribution of $\vec{\alpha}^{(i)}$ should follow the IEEE 802.15.4a channel model.

\section{ZIV-ZAKAI LOWER BOUND FOR IR-UWB RANGING ERROR CORRELATION MATRIX}

The estimation error of ranging vector is $\vec{\epsilon}=\hat{\vec{d}}-$ $\vec{d}$, where $\hat{\vec{d}}$ is the estimation of $\vec{d}$. The ranging error correlation matrix is defined as $\mathbf{R}_{\vec{\epsilon}}=E_{\vec{r}^{(s)}, \boldsymbol{\Theta}_{1}, \vec{d}}\{\vec{\epsilon}$. $\left.\vec{\epsilon}^{T}\right\}$, where $\vec{r}^{(s)}=\left[\vec{r}^{(1)^{T}} \cdots \vec{r}^{(I)^{T}}\right]^{T}$ and $\boldsymbol{\Theta}_{1}=$ $\left(\vec{\alpha}^{(1)}, \cdots, \vec{\alpha}^{(I)}, e^{(1)}, \cdots, e^{(I)}, T_{\eta}\right)$. We are interested in lower-bounding $\zeta=\vec{a}^{T} \mathbf{R}_{\vec{\epsilon}} \vec{a}$ for any $I$-dimensional vector $\vec{a}$. We can obtain the bounds on the elements of $\mathbf{R}_{\vec{\epsilon}}$ by setting different values of $\vec{a}$.

As $\vec{d}=\vec{g} \cdot c \cdot T_{s}, \zeta=\zeta_{g} \cdot c^{2} \cdot T_{s}^{2}$, where $\zeta_{g}=\vec{a}^{T} \mathbf{R}_{\vec{\epsilon}_{g}} \vec{a}$, $\mathbf{R}_{\vec{\epsilon}_{g}}=E_{\vec{r}(s), \Theta_{1}, \vec{g}}\left\{\vec{\epsilon}_{g} \vec{\epsilon}_{g}^{T}\right\}$ and $\vec{\epsilon}_{g}=\hat{\vec{g}}-\vec{g} . \hat{\vec{g}}$ is the estimation of $\vec{g}$. To find the lower bound of $\zeta$, we can compute the lower bound of $\zeta_{g}$ first. $\zeta_{g}$ can be computed as follows

$$
\zeta_{g}=E_{\vec{r}^{(s)}, \Theta_{1}, \vec{g}}\left\{\left|\vec{a}^{T} \vec{\epsilon}_{g}\right|^{2}\right\}=\int \zeta_{1} p\left(\boldsymbol{\Theta}_{1}\right) \mathrm{d} \boldsymbol{\Theta}_{1},
$$

where $\zeta_{1}=\iint\left|\vec{a}^{T} \vec{\epsilon}_{g}\right|^{2} p\left(\vec{r}^{(s)}, \vec{g} \mid \mathbf{\Theta}_{1}, \boldsymbol{\Theta}_{2}\right) \mathrm{d} g \mathrm{~d} \vec{r}$. and $\boldsymbol{\Theta}_{2}=$ $\left(\sigma^{(1)}, \cdots, \sigma^{(I)}\right)$.

To find the lower bound of $\zeta_{g}$, we need to find the lower bound of $\zeta_{1}$ first. Then, we average the lower bound of $\zeta_{1}$ over $\boldsymbol{\Theta}_{1}$.

The derivation starts from the identity (in the page 34 of [8]) as follows

$$
\zeta_{1}=\frac{1}{2} \int_{0}^{\infty} p\left(\left|\vec{a}^{T} \vec{\epsilon}_{g}\right| \geq \frac{h}{2} \mid \boldsymbol{\Theta}_{1}, \boldsymbol{\Theta}_{2}\right) h \mathrm{~d} h .
$$

The lower bound of $\zeta_{1}$ can be obtained by lower-bounding $p\left(\left|\vec{a}^{T} \vec{\epsilon}_{g}\right| \geq \frac{h}{2} \mid \boldsymbol{\Theta}_{1}, \boldsymbol{\Theta}_{2}\right)$ since $h$ and $p\left(\left|\vec{a}^{T} \vec{\epsilon}_{g}\right| \geq \frac{h}{2} \mid \boldsymbol{\Theta}_{1}, \boldsymbol{\Theta}_{2}\right)$ 
are nonnegative.

$$
\begin{aligned}
& p\left(\left|\vec{a}^{T} \vec{\epsilon}_{g}\right| \geq \frac{h}{2} \mid \boldsymbol{\Theta}_{1}, \boldsymbol{\Theta}_{2}\right)=p\left(\vec{a}^{T}(\hat{\vec{g}}-\vec{g})>\frac{h}{2} \mid \boldsymbol{\Theta}_{1}, \boldsymbol{\Theta}_{2}\right) \\
& +p\left(\vec{a}^{T}(\hat{\vec{g}}-\vec{g}) \leq-\frac{h}{2} \mid \boldsymbol{\Theta}_{1}, \boldsymbol{\Theta}_{2}\right) \\
& =\sum_{\vec{\varphi}_{0} \in \mathbf{G}} p\left(\vec{a}^{T}(\hat{\vec{g}}-\vec{g})>\frac{h}{2} \mid \boldsymbol{\Theta}_{1}, \boldsymbol{\Theta}_{2}, \vec{g}=\vec{\varphi}_{0}\right) p\left(\vec{g}=\vec{\varphi}_{0}\right) \mathrm{d} \vec{\varphi}_{0} \\
& +\sum_{\overrightarrow{\varphi_{1}} \in \mathbf{G}} p\left(\vec{a}^{T}(\hat{\vec{g}}-\vec{g}) \leq-\frac{h}{2} \mid \boldsymbol{\Theta}_{1}, \boldsymbol{\Theta}_{2}, \vec{g}=\vec{\varphi}_{1}\right) p\left(\vec{g}=\vec{\varphi}_{1}\right) \mathrm{d} \vec{\varphi}_{1},
\end{aligned}
$$

where $\mathbf{G}$ is the set that contains all possible values of $\vec{g}$.

We assume $\vec{\varphi}_{0}=\vec{\varphi}$ and $\vec{\varphi}_{1}=\vec{\varphi}+\vec{\delta}$, then multiplying and dividing $p(\vec{g}=\vec{\varphi})+p(\vec{g}=\vec{\varphi}+\vec{\delta})$ gives

$$
\begin{aligned}
& p\left(\left|\vec{a}^{T} \vec{\epsilon}_{g}\right| \geq \frac{h}{2} \mid \boldsymbol{\Theta}_{1}, \boldsymbol{\Theta}_{2}\right)=\sum_{\vec{\varphi} \in \mathbf{G}}(p(\vec{g}=\vec{\varphi})+p(\vec{g}=\vec{\varphi}+\vec{\delta})) \\
& \cdot\left[\frac{p(\vec{g}=\vec{\varphi})}{p(\vec{g}=\vec{\varphi})+p(\vec{g}=\vec{\varphi}+\vec{\delta})} p\left(\vec{a}^{T} \hat{\vec{g}}>\vec{a}^{T} \vec{\varphi}+\frac{h}{2} \mid \boldsymbol{\Theta}_{1}, \boldsymbol{\Theta}_{2}, \vec{g}=\vec{\varphi}\right)\right. \\
& \left.+\frac{p(\vec{g}=\vec{\varphi}+\vec{\delta})}{p(\vec{g}=\vec{\varphi})+p(\vec{g}=\vec{\varphi}+\vec{\delta})} p\left(\vec{a}^{T} \hat{\vec{g}} \leq \vec{a}^{T} \vec{\varphi}+\vec{a}^{T} \vec{\delta}-\frac{h}{2} \mid \Theta_{1}, \Theta_{2}, \vec{g}=\vec{\varphi}+\vec{\delta}\right)\right] \mathrm{d} \vec{\varphi} .
\end{aligned}
$$

For a given $h$, if $\vec{\delta}$ is chosen to satisfy

$$
\vec{a}^{T} \vec{\delta}=h \text { and } \vec{\varphi}+\delta \in \mathbf{G}
$$

then Eq.(4) becomes

$$
\begin{aligned}
& p\left(\left|\vec{a}^{T} \vec{\epsilon}_{g}\right| \geq \frac{h}{2} \mid \boldsymbol{\Theta}_{1}, \boldsymbol{\Theta}_{2}\right)=\sum_{\vec{\varphi} \in \mathbf{G}}(p(\vec{g}=\vec{\varphi})+p(\vec{g}=\vec{\varphi}+\vec{\delta})) \\
& \cdot\left[\frac{p(\vec{g}=\vec{\varphi})}{p(\vec{g}=\vec{\varphi})+p(\vec{g}=\vec{\varphi}+\vec{\delta})} p\left(\vec{a}^{T} \hat{\vec{g}}>\vec{a}^{T} \vec{\varphi}+\frac{h}{2} \mid \boldsymbol{\Theta}_{1}, \boldsymbol{\Theta}_{2}, \vec{g}=\vec{\varphi}\right)\right. \\
& \left.+\frac{p(\vec{g}=\vec{\varphi}+\vec{\delta})}{p(\vec{g}=\vec{\varphi})+p(\vec{g}=\vec{\varphi}+\vec{\delta})} p\left(\vec{a}^{T} \hat{\vec{g}} \leq \vec{a}^{T} \vec{\varphi}+\frac{h}{2} \mid \boldsymbol{\Theta}_{1}, \boldsymbol{\Theta}_{2}, \vec{g}=\vec{\varphi}+\vec{\delta}\right)\right] \mathrm{d} \vec{\varphi}
\end{aligned}
$$

Now consider the detection problem defined by

$$
\begin{array}{ll}
\mathbf{H}_{\mathbf{0}}: \vec{g}=\vec{\varphi} ; & \vec{r} \sim p\left(\vec{r} \mid \boldsymbol{\Theta}_{1}, \boldsymbol{\Theta}_{2}, \vec{\varphi}\right), \\
\mathbf{H}_{\mathbf{1}}: \vec{g}=\vec{\varphi}+\vec{\delta} ; & \vec{r} \sim p\left(\vec{r} \mid \boldsymbol{\Theta}_{1}, \boldsymbol{\Theta}_{2}, \vec{\varphi}+\vec{\delta}\right),
\end{array}
$$

with $p\left(\mathbf{H}_{\mathbf{0}}\right)=\frac{p(\vec{g}=\vec{\varphi})}{p(\vec{g}=\vec{\varphi})+p(\vec{g}=\vec{\varphi}+\vec{\delta})}$, where we can find that $p\left(\mathbf{H}_{\mathbf{1}}\right)=1-p\left(\mathbf{H}_{\mathbf{0}}\right)$.

the values of $p(\vec{g}=\vec{\varphi})$ and $p(\vec{g}=\vec{\varphi}+\vec{\delta})$ through the histogram of $\vec{g}$ when the distribution of $\vec{g}$ depend on the geometry of the indoor environments.

The suboptimal decision rule in which the parameter is first estimated and a decision is made in favor of its "nearest neighbor"

$$
\begin{array}{ll}
\text { Decide } \mathbf{H}_{\mathbf{0}}: \vec{g}=\vec{\varphi}, & \text { if } \vec{a}^{T} \hat{\vec{g}} \leq \vec{a}^{T} \vec{\varphi}+\frac{h}{2}, \\
\text { Decide } \mathbf{H}_{\mathbf{1}}: \vec{g}=\vec{\varphi}+\vec{\delta}, & \text { if } \vec{a}^{T} \hat{\vec{g}}>\vec{a}^{T} \vec{\varphi}+\frac{h}{2} .
\end{array}
$$

The term in square bracket in Eq.(5) is the probability of error for this suboptimal decision scheme. It can be lowerbounded by the minimum probability of error obtained from the likelihood ratio test $P_{\min }\left(\vec{g}=\vec{\varphi}, \vec{g}=\vec{\varphi}+\vec{\delta} \mid \Theta_{1}, \boldsymbol{\Theta}_{2}\right)$. Therefore,

$$
\begin{aligned}
& p\left(\left|\vec{a}^{T} \vec{\epsilon}_{g}\right| \geq \frac{h}{2} \mid \boldsymbol{\Theta}_{1}, \boldsymbol{\Theta}_{2}\right) \geq \sum_{\vec{\varphi} \in \mathbf{G}}(p(\vec{g}=\vec{\varphi})+p(\vec{g}=\vec{\varphi}+\vec{\delta})) \\
& \cdot P_{\min }\left(\vec{g}=\vec{\varphi}, \vec{g}=\vec{\varphi}+\vec{\delta} \mid \boldsymbol{\Theta}_{1}, \boldsymbol{\Theta}_{2}\right) .
\end{aligned}
$$

The decision based on the likelihood ratio test is made according to the following rule:

$$
\begin{array}{ll}
\text { Decide } \mathbf{H}_{\mathbf{0}}: \vec{g}=\vec{\varphi}, & \text { if } \Lambda\left(\vec{r}^{(s)}\right)>\Delta_{\vec{\varphi}, \vec{\delta}} \\
\text { Decide } \mathbf{H}_{\mathbf{1}}: \vec{g}=\vec{\varphi}+\vec{\delta}, & \text { if } \Lambda\left(\vec{r}^{(s)}\right) \leq \Delta_{\vec{\varphi}, \vec{\delta}}
\end{array}
$$

where $\Lambda\left(\vec{r}^{(s)}\right)=\frac{p\left(\vec{r}^{(s)} \mid \vec{g}=\vec{\varphi}, \boldsymbol{\Theta}_{1}, \boldsymbol{\Theta}_{2}\right)}{p\left(\vec{r}^{(s)} \mid \vec{g}=\vec{\varphi}+\vec{\delta}, \boldsymbol{\Theta}_{1}, \boldsymbol{\Theta}_{2}\right)}$, and $\Delta_{\vec{\varphi}, \vec{\delta}}=\frac{p\left(\mathbf{H}_{1}\right)}{p\left(\mathbf{H}_{\mathbf{0}}\right)}=$ $\frac{p(\vec{g}=\varphi+\vec{\delta})}{p(\vec{g}=\vec{\varphi})}$. lows

The $\log$-likelihood $\log \left\{\Lambda\left(\vec{r}^{(s)}\right)\right\}$ can be computed as fol-

$$
\begin{aligned}
& \log \left\{\Lambda\left(\vec{r}^{(s)}\right)\right\}=\log \left\{\frac{\prod_{i=1}^{I} p\left(\vec{r}^{(i)} \mid \vec{g}=\vec{\varphi}, \vec{\alpha}^{(i)}, \eta, \sigma^{(i)}\right)}{\prod_{i=1}^{I} p\left(\vec{r}^{(i)} \mid \vec{g}=\vec{\varphi}+\vec{\delta}, \vec{\alpha}^{(i)}, \eta, \sigma^{(i)}\right)}\right\} \\
& =\sum_{i=1}^{I}\left\{-\frac{1}{2\left(\sigma^{(i)}\right)^{2}} \cdot \sum_{k=0}^{K-1}\left[\mathbb{B}^{(i)}(k)+2 \mathbb{A}^{(i)}(k) r^{(i)}(k)\right]\right\} .
\end{aligned}
$$

Given $\vec{g}=\vec{\varphi}$ and $\Theta_{1}, \rho_{n, \vec{\varphi}}^{(i)}(k)$ can be computed according to Eq.(1). Given $\vec{g}=\vec{\varphi}+\vec{\delta}$ and $\Theta_{1}, \rho_{n, \vec{\varphi}+\vec{\delta}}^{(i)}(k)$ can be computed according to Eq.(1). And also, $\mathbb{A}^{(i)}(k)=\rho_{n, \vec{\varphi}+\vec{\delta}}^{(i)}(k)-\rho_{n, \vec{\varphi}}^{(i)}(k)$, and $\mathbb{B}^{(i)}(k)=\left(\rho_{n, \vec{\varphi}}^{(i)}(k)\right)^{2}-\left(\rho_{n, \vec{\varphi}+\vec{\delta}}^{(i)}(k)\right)^{2}$.

If $\log \left\{\Lambda\left(\vec{r}^{(s)}\right)\right\}>\log \Delta_{\vec{\varphi}, \vec{\delta}}$, we can decide $\mathbf{H}_{\mathbf{0}}$ and obtain

where $\chi=\sum_{i=1}^{I}\left\{\frac{1}{\left(\sigma^{(i)}\right)^{2}} \sum_{k=0}^{K-1} \mathbb{A}^{(i)}(k) r^{(i)}(k)\right\}$, and $\mathbb{C}=$ $-\sum_{i=1}^{I}\left\{\frac{1}{2\left(\sigma^{(i)}\right)^{2}} \sum_{k=0}^{K-1} \mathbb{B}^{(i)}(k)\right\}-\log \Delta_{\vec{\varphi}, \vec{\delta}}$.

$$
\begin{aligned}
& r^{(i)}(k) \sim \mathcal{N}\left(\rho_{\vec{\varphi}}^{(i)}(k),\left(\sigma^{(i)}\right)^{2}\right) ; \quad \text { conditioned on } \mathbf{H}_{\mathbf{0}}, \\
& r^{(i)}(k) \sim \mathcal{N}\left(\rho_{\vec{\varphi}+\vec{\delta}}^{(i)}(k),\left(\sigma^{(i)}\right)^{2}\right) ; \quad \text { conditioned on } \mathbf{H}_{\mathbf{1}} \text {, }
\end{aligned}
$$

where $\rho_{\vec{\varphi}}^{(i)}(k)=\rho_{n, \vec{\varphi}}^{(i)}(k)$ and $\rho_{\vec{\varphi}+\vec{\delta}}^{(i)}(k)=\rho_{n, \vec{\varphi}+\vec{\delta}}^{(i)}(k)$.

Therefore, the distribution of $\chi$ is given as follows

$$
\begin{array}{ll}
\chi \sim \mathcal{N}\left(\mu_{\vec{\varphi}}, \sigma_{\chi}^{2}\right) ; & \text { conditioned on } \mathbf{H}_{\mathbf{0}}, \\
\chi \sim \mathcal{N}\left(\mu_{\vec{\varphi}+\vec{\delta}}, \sigma_{\chi}^{2}\right) ; & \text { conditioned on } \mathbf{H}_{\mathbf{1}},
\end{array}
$$

where

$$
\begin{gathered}
\mu_{\vec{\varphi}}=\sum_{i=1}^{I} \sum_{k=0}^{K-1} \frac{\mathbb{A}^{(i)}(k) \rho_{\vec{\varphi}}^{(i)}(k)}{E^{(i)}} \cdot \mathrm{SNR}^{(i)}, \\
\mu_{\vec{\varphi}+\vec{\delta}}=\sum_{i=1}^{I} \sum_{k=0}^{K-1} \frac{\mathbb{A}^{(i)}(k) \rho_{\vec{\varphi}+\vec{\delta}}^{(i)}(k)}{E^{(i)}} \cdot \mathrm{SNR}^{(i)}, \\
\sigma_{\chi}^{2}=\sum_{i=1}^{I} \sum_{k=0}^{K-1} \frac{\left(\mathbb{A}^{(i)}(k)\right)^{2}}{E^{(i)}} \mathrm{SNR}^{(i)}
\end{gathered}
$$

and the Signal to Noise Ratio (SNR) of the $i$-th base station is defined as $\mathrm{SNR}^{(i)}=\frac{E^{(i)}}{\left(\sigma^{(i)}\right)^{2}}$, where $E^{(i)}$ is the received energy per pulse (after the convolution of the pulse with the impulse response of the channel).

Finally, $P_{\min }\left(\vec{g}=\vec{\varphi}, \vec{g}=\vec{\varphi}+\vec{\delta} \mid \Theta_{1}, \Theta_{2}\right)$ can be evaluated as follows

$$
\begin{aligned}
& P_{\min }\left(\vec{g}=\vec{\varphi}, \vec{g}=\vec{\varphi}+\vec{\delta} \mid \boldsymbol{\Theta}_{1}, \mathbf{\Theta}_{2}\right)=p\left(\mathbf{H}_{\mathbf{0}} \mid \mathbf{H}_{\mathbf{1}}\right) p\left(\mathbf{H}_{\mathbf{1}}\right) \\
+ & p\left(\mathbf{H}_{\mathbf{1}} \mid \mathbf{H}_{\mathbf{0}}\right) p\left(\mathbf{H}_{\mathbf{0}}\right) \\
= & p\left(\chi<\mathbb{C} \mid \mathbf{H}_{\mathbf{1}}\right) \cdot p\left(\mathbf{H}_{\mathbf{1}}\right)+p\left(\chi \geq \mathbb{C} \mid \mathbf{H}_{\mathbf{0}}\right) \cdot p\left(\mathbf{H}_{\mathbf{0}}\right) \\
= & \left(1-\mathbb{Q}\left(\frac{\mathbb{C}-\mu_{\vec{\varphi}+\vec{\delta}}}{\sqrt{\sigma_{\chi}^{2}}}\right)\right) \cdot p\left(\mathbf{H}_{\mathbf{1}}\right)+\mathbb{Q}\left(\frac{\mathbb{C}-\mu_{\vec{\varphi}}}{\sqrt{\sigma_{\chi}^{2}}}\right) \cdot p\left(\mathbf{H}_{\mathbf{0}}\right),
\end{aligned}
$$

where $\mathbb{Q}(\cdot)$ is the complementary cumulative distribution function.

Given $\Theta_{1}$ and $\Theta_{2}$, the lower bound of $\zeta_{1}$ can be computed according to Eq.(3), Eq.(6) and Eq.(7):

$$
\begin{aligned}
& \zeta_{1} \geq \zeta_{2}=\frac{1}{2} \int_{0}^{\infty} \sum_{\vec{\varphi} \in \mathbf{G}}(p(\vec{g}=\vec{\varphi})+p(\vec{g}=\vec{\varphi}+\vec{\delta})) \\
& \cdot P_{\min }\left(\vec{g}=\vec{\varphi}, \vec{g}=\vec{\varphi}+\vec{\delta} \mid \mathbf{\Theta}_{1}, \boldsymbol{\Theta}_{2}\right) h \mathrm{~d} h .
\end{aligned}
$$




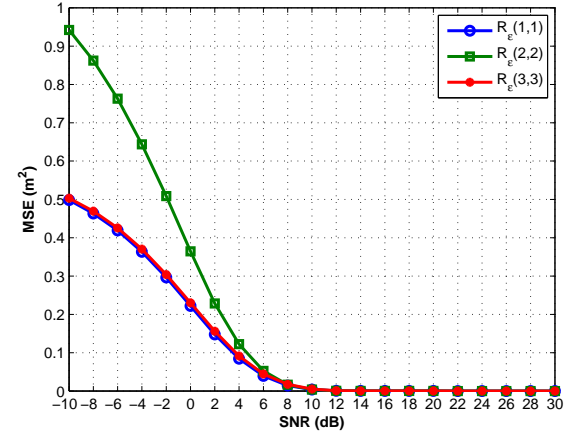

(a)

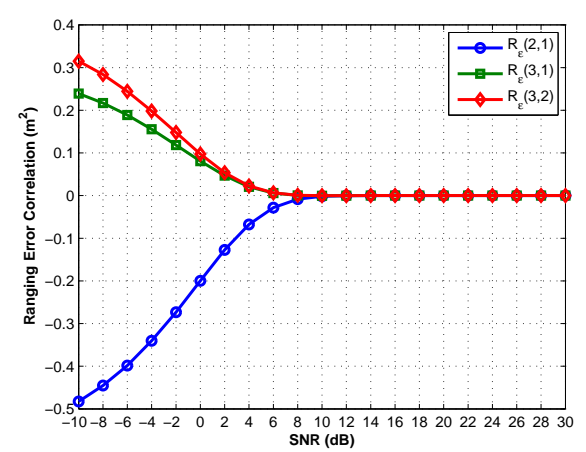

(b)

Fig. 3. (a) The Ziv-Zakai lower bound of diagonal elements of $\mathbf{R}_{\vec{\epsilon}}$. (b) The Ziv-Zakai lower bound of the non-diagonal elements of $\mathbf{R}_{\vec{\epsilon}}$.

According to Eq.(2), the Ziv-Zakai lower bound of $\zeta_{g}$ and $\zeta$ can be evaluated by using Monte Carlo simulations. We can draw $N_{5}$ samples of $\boldsymbol{\Theta}_{1}$ from their corresponding distributions. For each sample of $\boldsymbol{\Theta}_{1, n_{5}}$, we can compute one value of $\zeta_{2}$ according to Eq.(8) (where $n_{5} \in\left\{0, \cdots, N_{5}-1\right\}$ ). Therefore, the Ziv-Zakai lower bound of $\zeta$ can be computed as follows

$$
\begin{aligned}
& \zeta \geq\left\{\frac{1}{N_{5}} \sum_{n_{5}=0}^{N_{5}-1} \zeta_{2, n_{5}}\right\} \cdot c^{2} \cdot T_{s}^{2} . \\
& \text { V. NuMERICAL EVALUATIONS }
\end{aligned}
$$

\section{Numerical Evaluations}

The transmitted pulse $f(t)$ is selected as the second derivative of the Gaussian function with duration $T_{f}=1 \mathrm{~ns}$ that is a typical duration of an IR-UWB pulse. The bandwidth of the band-pass filter in the receiver is $500 \mathrm{MHz}$. The sampling frequency is $2 \mathrm{GHz}$. We use 100 channel realizations to compute the Ziv-Zakai lower bound for IR-UWB ranging error correlation matrix. We use IEEE 802.15.4a CM1 channel model as the LOS channel. We use IEEE 802.15.4a CM2 channel model as the NLOS channel. The TOA of the first path of IEEE 802.15.4a CM2 channel model is set to zero. We use the samples of $e^{(i)}$ as the NLOS delay. The probability of $0.5 m \leq e^{(i)} \leq 1.2 m$ is equal to 0.7 . The probability of $1.5 m \leq e^{(i)} \leq 2.1 m$ is equal to 0.3 . The values of all base stations' SNR are the same. The mobile tag is in a rectangular room with furniture (as shown in Fig.2.)

We assume the number of the base stations is 3. Therefore, $\mathbf{R}_{\vec{\epsilon}}$ is a $3 \times 3$ matrix. We denote $\mathbf{R}_{\vec{\epsilon}}(i, j)$ as the element in $i$-th row and $j$-th column. The position of the base stations are $\left(x^{(1)}=-3, y^{(1)}=0\right),\left(x^{(2)}=3, y^{(2)}=0\right)$ and $\left(x^{(3)}=\right.$ $\left.0, y^{(1)}=3\right)$. We assume that the channel between the 1 -th base station and the mobile tag is a NLOS channel, the other two channels between the mobile tag and the base stations are LOS channels.

In Fig.3(a), we show the Ziv-Zakai lower bound of the diagonal elements of $\mathbf{R}_{\vec{\epsilon}} \cdot \mathbf{R}_{\vec{\epsilon}}(i, i)$ is the Ziv-Zakai lower bound of the ranging error between the mobile tag and $i$-th base station. We find that the Ziv-Zakai lower bound of ranging error decreases as the SNR increases. We also find that the Ziv-Zakai lower bounds of the ranging errors with different base stations are different. This is due to the prior distributions of the ranging with different base stations are different.

In Fig.3(b), we show the Ziv-Zakai lower bound of the non-diagonal elements of $\mathbf{R}_{\vec{\epsilon}}$. $\mathbf{R}_{\vec{\epsilon}}(i, j)$ shows the Ziv-Zakai lower bound of the ranging error correlation between $i$-th base station and $j$-th base station. We find that the ranging error between the different base stations are correlated. We find that the Ziv-Zakai lower bound of the ranging errors correlation approximate to 0 when SNR increases.

\section{CONCLUSIONS}

We have derived the Ziv-Zakai lower bound for IR-UWB ranging error correlation matrix under a classical IR-UWB positioning system. We present the numerical evaluations with IEEE 802.15.4a channel model and the geometry of indoor environments of interest. As our derived bound depends on the geometry of the indoor environments, our bound can be used in real environments with the channel measurements from real environments.

\section{REFERENCES}

[1] R. Fontana and S. Gunderson, "Ultra-wideband precision asset location system," Ultra Wideband Systems and Technologies, 2002. Digest of Papers. 2002 IEEE Conference on, pp. 147-150, 2002.

[2] J. Zhang, R. Kennedy, and T. Abhayapala, "Cramer-rao lower bounds for the time delay estimation of uwb signals," in Communications, 2004 IEEE International Conference on, vol. 6, June 2004, pp. 3424-3428 Vol.6.

[3] K. Bell, Y. Steinberg, Y. Ephraim, and H. Van Trees, "Extended ziv-zakai lower bound for vector parameter estimation," Information Theory, IEEE Transactions on, vol. 43, no. 2, pp. 624-637, Mar 1997.

[4] H. Anouar, A. Hayar, R. Knopp, and C. Bonnet, "Lower bound on time-delay estimation error of uwb signals," in Signals, Systems and Computers, 2007. ACSSC 2007. Conference Record of the Forty-First Asilomar Conference on, Nov. 2007, pp. 1350-1354.

[5] D. Dardari, C.-C. Chong, and M. Win, "Improved lower bounds on timeof-arrival estimation error in realistic uwb channels," in Ultra-Wideband, The 2006 IEEE 2006 International Conference on, Sept. 2006, pp. 531537.

[6] J. Boudec, Performance Evaluation. [Online] Availabe: http://ica1www.epfl.ch/perfeval/documents.htm, 2006.

[7] D. Jourdan, D. Dardari, and M. Win, "Position error bound for uwb localization in dense cluttered environments," Aerospace and Electronic Systems, IEEE Transactions on, vol. 44, no. 2, pp. 613-628, April 2008.

[8] E. Cinlar, Introduction to Stochastic Processes. Englewood Cliffs, NJ:Prentice-Hall. 